\title{
Validation of Neutron Evaluated Data Based on The Experimental Reactivity Worth of Tungsten Target in CiADS
}

\author{
Wei Jiang ${ }^{1,2}$, Long $\mathrm{Gu}^{1,2^{*}}$, Lu Zhang ${ }^{1}$, Qi Zhou ${ }^{3}$, Liang Chen ${ }^{1}$, \\ Jin-Yang $\mathrm{Li}^{1}$, Rui $\mathrm{Yu}^{1}$, Qing-Fu Zhu ${ }^{3}$, Yong Dai ${ }^{2}$ \\ ${ }^{1}$ Institute of Modern Physics, Chinese Academy of Sciences, Lanzhou, 730000, China \\ ${ }^{2}$ Paul Scherrer Institute, Villigen, 5232, Switzerland \\ ${ }^{3}$ China Institute of Atomic Energy, China National Nuclear Corporation, Beijing, 102413, China \\ *gulong@impcas.ac.cn
}

\begin{abstract}
Measurement of a cylindrical tungsten target reactivity worth has been performed on the light water zero-power reactor of VENUS-II at China Institute of Atomic Energy (CIAE) in order to verify the neutron evaluated data related to the engineering design of Chinese initiative Accelerator Driven Systems (CiADS). The reactivity worth of the tungsten target was measured and processed as $-1.234 \pm 0.114 \mathrm{mk}$ by a period method. The experimental result was compared with the simulation ones calculated by MCNP with five different libraries, i.e., ENDF/B-VII.0, ENDF/B-VII.1, JENDL-4.0, CENDL-3.1 and JEFF-3.2. By comparing the results of experiment and simulation, the simulated results from ENDF/B-VII.0, JENDL-4.0 and JEFF-3.2 are higher than the experimental result, however that from CENDL-3.1 is lower. The result from ENDF/B-VII.1 library shows better agreement with the experiment one and the relative deviation is less than $2 \%$. Through the analysis of the differences of the results, non-tungsten elements cross sections in the ENDF/B-VII.1 mainly affect the tungsten radiation capture and elastic scattering reaction rates in the energy range of $10^{-9}-10^{-7} \mathrm{MeV}$, which results in a better simulated tungsten target reactivity worth value. Therefore, it is recommended that the tungsten target reactivity worth should be calculated with the ENDF/B-VII.1.

Keywords-Tungsten target reactivity worth, nuclear data library, MCNP, VENUS-II, period method
\end{abstract}

\section{INTRODUCTION}

Recently, Chinese initiative Accelerator Driven Systems (CiADS) project has been started in China [1]. For CiADS, the primary goal is to achieve the integration of three systems, i.e. a subcritical lead-bismuth reactor, a heavy metal spallation target and an intense-beam proton linac [2]. During the early stages of ADS projects, some experiments [3-7], which coupled the proton accelerator with the spallation target, have been performed by the researchers. However, the experimental studies about the coupling behaviors between the nuclear reactor and the spallation target have been rarely investigated. In order to ultimately build the CiADS experimental setup, it is necessary that the nuclear reactor and spallation target coupling experiments should be performed. The tungsten granular spallation target has been proposed by Institute of Modern Physics, Chinese Academy of Sciences (IMPCAS) as an innovative spallation target in the world. The tungsten granular target, which is placed in the center of the reactor, is impinged by the high-energy protons to produce spallation neutrons to maintain the stable operation of the system [8]. Meanwhile, the spallation target, as the structural material, has an effect on the reactor physical parameters, such as neutron flux, neutron spectra, system reactivity and so on. The mass fraction of tungsten is $93 \%$ in the spallation target, therefore tungsten cross sections in evaluated data libraries can impact directly on the accuracy of reactor physical calculations in CiADS. In the past experiment on tungsten data evaluation, tungsten materials are generally used as reflecting layer or fuel diluent materials and almost placed in fast-spectrum benchmark experiment setups [9-15]. However, the relevant studies on the tungsten spallation materials in thermal-spectrum reactor are still scarce. In this paper, the measurement of the cylindrical tungsten target reactivity worth was performed on VENUS-II light water reactor by the period method. And then the experimental tungsten target reactivity worth was compared with the results calculated by MCNP with the different libraries, i.e. ENDF/B-VII.0 [16], ENDF/B-VII.1 [17], JENDL-4.0 [18], JEFF-3.2 [19] and CENDL-3.1 [20]. The difference between the experimental and simulated results is discussed, which provides some reference for the selection of nuclear data libraries on CiADS neutronics simulations.

\section{EXPERIMENT}

II.1 Experimental facility

The VENUS-II zero-power facility, which consists of a light water reactor and a lead reactor, firstly reached the criticality on December 2016 through the cooperation of China Institute of Atomic Energy (CIAE) and Institute of Modern Physics, Chinese Academy of Sciences [21]. The structures and materials in the VENUS-II light water reactor are relatively simple, which is helpful in validating the neutron nuclear data libraries. Therefore, the present experiments were carried out on the light water reactor. The physical photos of VENUS-II light water reactor are shown in Fig. 1. Light water was used as the moderator and reflector, in which eight $\gamma$ compensation ionization chambers were arranged to monitor nuclear reactor power. Fuel rods of $\mathrm{U}_{3} \mathrm{O}_{8}$ with the enrichment $20 \%$ were arranged outside the spallation zone of $\phi 170 \mathrm{~mm} \times \mathrm{H} 804 \mathrm{~mm}$ in the concentric manner. The experimental tungsten target, with the dimensions of $\phi 100 \mathrm{~mm} \times \mathrm{H} 100 \mathrm{~mm}$, was placed on the bottle of the spallation target zone in the center of the core. The tungsten target purity and density were respectively $99.95 \%$ and $18.2 \mathrm{~g} / \mathrm{cm}^{3}$. The 304 stainless steel target clad with the thickness of $2 \mathrm{~mm}$ was helpful to place the cylindrical tungsten target in and out the spallation target zone.

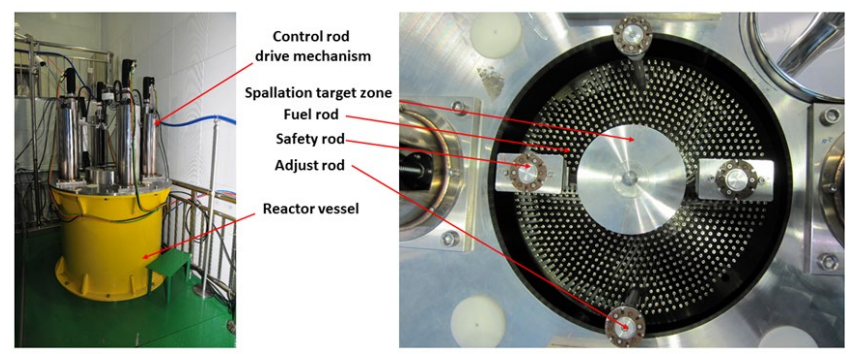

Fig. 1. Physical photos of VENUS-II light water reactor

\section{II.2 Experimental principle and data processing}

The reactivity $\rho$ values of the VENUS-II light water reactor are measured by the period method [22]. Base on the point reactor dynamics equation, in-hour equation is derived and as followed: 


$$
\rho=\frac{\Lambda}{\mathrm{T} / \ln 2}+\sum_{\mathrm{i}=1}^{\mathrm{m}} \frac{\beta_{\mathrm{ieff}}}{1+\left(\lambda_{i} T / \ln 2\right)}
$$

Where, $\rho$ is the reactivity; $\Lambda$ is the neutron generation time; $T$ is the nuclear reactor doubling time; $\mathrm{m}$ is the number of effective delayed neutron groups; $\beta_{i, \text { eff }}$ and $\lambda_{i}$ are respectively the ith group effective delayed neutron fraction and the decay constant of the ith group delayed neutron precursor.

The double period $\mathrm{T}$ values were measured by stopwatch and reactor power monitor [23] in different supercritical states of the reactor, and the supercritical states were maintained by adjusting the fuel rods number in the reactor. During the experiment, the four supercritical states to be measured are as follows: (1) 961 fuel rods without anything in the spallation zone, (2) 960 fuel rods without anything in the spallation zone, (3) 963 fuel rods with the target clad in the spallation zone, and (4) 968 fuel rods with tungsten target (include cylindrical tungsten target and target clad) in the spallation zone. More details on the experiments were reported in the Ref. [24]. Meanwhile, the reactor kinetic parameters $\left(\Lambda, \beta_{i, \text { eff }}\right.$ and $\left.\lambda_{i}\right)$ were respectively calculated by MCNP6 with different libraries, i.e., ENDF/B-VII.0, ENDF/B-VII.1, JENDL-4.0, CENDL-3.1 and JEFF-3.2. And then the reactivity $\rho$ values were calculated by substituting the measured $\mathrm{T}$ and the calculated kinetic parameters into the Eq. (1). At last, according to the formula $\Delta \rho=\rho_{968}-\left[\rho_{963}+5 \times\left(\rho_{961}-\rho_{960}\right)\right]$, the tungsten target reactivity worth $\Delta \rho$ was calculated as $-1.234 \pm 0.114 \mathrm{mk}$, where $\rho_{961}, \rho_{960}, \rho_{963}$ and $\rho_{968}$ represent the measured reactivity values under the above conditions (1)-(4), respectively. The experimental error comes from the deviations of $T$ measurement and the uncertainties of the simulated kinetic parameters [25].

\section{Monte Carlo Simulation}

\section{III.1 Simulation method}

In this paper, the reactivity worth of the cylindrical tungsten target is simulated by MCNP software, which is produced and developed by Los Alamos National Laboratory. In MCNP simulations, VENUS-II light water reactor model was created accurately at real geometry and material. Meanwhile, the loading number of fuel rods in the light water reactor was set as the number (totally 961 rods) of initial fuel rods in the experiment and kept the constant. And then the simulation process had two steps: (1) The target clad was placed in the spallation target zone; (2) The tungsten target replaced the target clad in the spallation target zone. Therefore, the reactivity worth of the tungsten target was calculated as the difference between the reactor reactivity values with the tungsten target and the target clad in the spallation target. The simulation method can reduce the simulated error of the tungsten target reactivity worth results and satisfy the accuracy demands.

III.2 Details description

The effective multiplication factor keff values of the two steps in Part 3.1 were calculated by KCODE card in MCNP with 3000 cycles and $5^{*} 10^{5}$ histories per cycle, which caused the estimated standard deviation of about $2 \mathrm{pcm}\left(1 \mathrm{pcm}=10^{-5}\right)$ for the $\mathrm{k}_{\mathrm{eff}}$ results. The temperature values of the material cross sections were set as the room temperature $293 \mathrm{k}$ and remained unchanged. Considering the upward scattering of the thermal neutrons, the thermal neutron scattering library $\mathrm{S}(\alpha, \beta)$ was used to achieve the accurate neutron transportation calculations in the light water reactor. Then, the keff was converted to the reactivity $\rho$ through the equation $\rho=\left(k_{\text {eff }}-1\right) / k_{\text {eff }}$. The simulated tungsten target reactivity worth was calculated as the difference between the reactivity values with the tungsten target and the target clad in the spallation zone.

To examine the different neutron nuclear data libraries, the cross sections of all materials in the reactor, i.e. target, fuels, moderator and among others, are respectively from ENDF/B-VII.0, ENDF/B-VII.1, CENDL-3.1, JENDL-4.0 and JEFF-3.2. And then five corresponding tungsten reactivity worth values were obtained and the errors originated from the statistical errors of MCNP simulation.

\section{RESULTS AND DISCUSSION}

The experimental and calculated results of tungsten target reactivity worth values are shown in Fig. 2, where the shaded area indicates the experimental results within the error range, and the five dots respectively represent the MCNP simulated results with five cross section libraries. In Table I, the calculated results in the second column are respectively simulated by MCNP with the different cross section libraries. Meanwhile, the ratio values of the calculated and experimental results $(\mathrm{C} / \mathrm{E})$ are presented in the fourth column. From the obtained results, it can be observed that:

(1). Tungsten target reactivity worth values, obtained by the experiment and simulation, are all negative, which causes the reduction of the reactor reactivity.

(2). Comparing with the experimental result, the simulated results of ENDF/B-VII.0, JENDL-4.0 and JEFF-3.2 are higher, whereas the one of CENDL-3.1 is lower.

(3). The simulated result with ENDF/B-VII.1 has a good agreement with the experiment one and the relative deviation is less than $2 \%$.

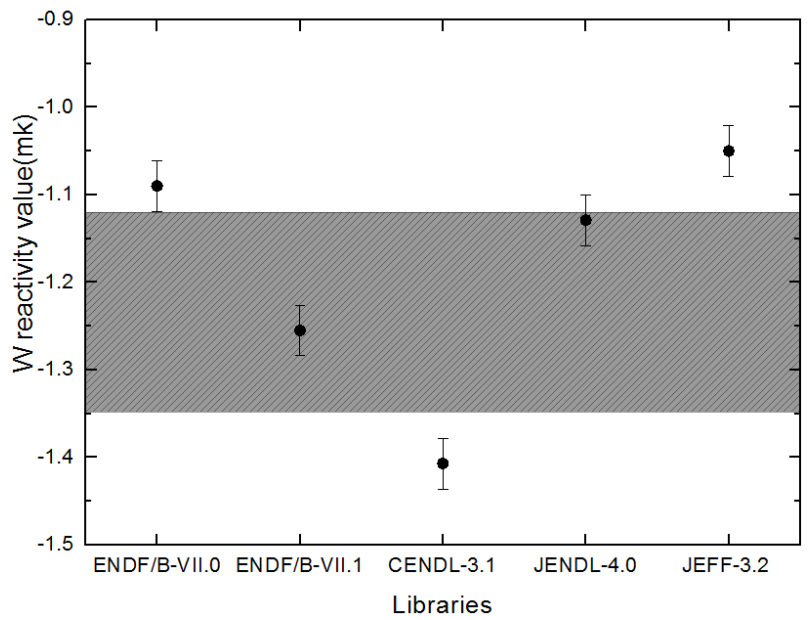

Fig. 2. Tungsten target reactivity worth values from both the experiment and the simulation.

TABLE I

TUNGSTEN TARGET REACTIVITY WORTH VALUES BETWEEN THE MCNP CALCULATIONS AND PERIOD METHOD

\begin{tabular}{|c|c|c|c|}
\hline \multirow{2}{*}{ Libraries } & \multicolumn{2}{|c|}{ Tungsten target reactivity worth (mk) } & \multirow{2}{*}{$\mathrm{C} / \mathrm{E}$} \\
\hline & MCNP & Period method & \\
\hline ENDF/B-VII.0 & $-1.090 \pm 0.029$ & & 0.883 \\
\hline ENDF/B-VII.1 & $-1.255 \pm 0.029$ & & 1.017 \\
\hline CENDL-3.1 & $-1.407 \pm 0.029$ & $-1.234 \pm 0.114$ & 1.14 \\
\hline JENDL-4.0 & $-1.129 \pm 0.029$ & & 0.915 \\
\hline JEFF-3.2 & $-1.050 \pm 0.029$ & & 0.851 \\
\hline
\end{tabular}


In order to find out the reason of the differences among the tungsten reactivity worth values from five libraries, the affecting factors of tungsten reactivity worth were divided into two aspects: the tungsten element and non-tungsten elements. Meanwhile, the simulation results from ENDF/B- VII.0, JENDL-4.0 and JEFF-3.2 are basically in agreement within the error ranges (see Fig. 2), so that the results are grouped into the same group and one calculated by ENDF/B-VII.0 was chosen to represent the group. Therefore, the simulated result from the $\mathrm{ENDF} / \mathrm{B}-\mathrm{VII} .1$ is only compared with those from the ENDF/B-VII.0 and CENDL-3.1. First, the reason for the difference between the tungsten reactivity worth results with the ENDF/B-VII.1 and CENDL-3.1 is mainly the discrepancy of the tungsten radiative capture cross sections at the epithermal neutron region [24].

Next, the study focused on analyzing the difference between the tungsten reactivity worth values from ENDF/B-VII.1 and ENDF/B-VII .0 . Therefore, the tungsten reactivity worth values in the three cases are calculated and shown in Table II. The table shows the results in the case 1 and case 2 are almost consistent with each other within errors. However, the result in case 1 is remarkably different from that in case 3 . It can be concluded that the non-tungsten element cross sections are the main reason for the discrepancy of the results between ENDF/BVII.0 and ENDF/B-VII.1, whereas the impact of the tungsten element cross sections on the difference is almost negligible.

$$
\text { TABLE II }
$$

THE SIMULATED TUNGSTEN TARGET REACTIVITY WORTH WITH NUCLEAR DATA IN THE ENDF/B-VII.0 AND ENDF/B-VII.1

\begin{tabular}{cccc}
\hline $\begin{array}{c}\text { Case } \\
\text { No. }\end{array}$ & $\begin{array}{c}\text { Tungsten } \\
\text { cross sections }\end{array}$ & $\begin{array}{c}\text { Non-tungsten } \\
\text { cross sections }\end{array}$ & Target worth (mk) \\
\hline 1 & ENDF/B-VII.1 & ENDF/B-VII.0 & $-1.110 \pm 0.029$ \\
2 & ENDF/B-VII.0 & ENDF/B-VII.0 & $-1.090 \pm 0.029$ \\
3 & ENDF/B-VII.1 & ENDF/B-VII.1 & $-1.255 \pm 0.029$ \\
\hline
\end{tabular}

In the neutron field of the reactor, the reaction channels in the tungsten target mainly include the radiative capture, elastic scattering, inelastic scattering, $(\mathrm{N}, 2 \mathrm{~N})$ and $(\mathrm{N}, 3 \mathrm{~N})$. To analyze the effect of the each reaction channel on the tungsten reactivity worth, the reaction rates of each reaction channel were respectively increased by 1.3 times the original values for the sensitivity analysis. More specifically, the input card in case 3 of Table II was calculated by the perturbation function of MCNP and the variations of the reactivity $\rho$ values are shown in Table III. For the specific reaction type, it can be seen that the radiative capture causes a negative reactivity in the system, whereas the elastic scattering and inelastic scattering respectively cause a positive reactivity. $(\mathrm{N}, 2 \mathrm{~N})$ and $(\mathrm{N}, 3 \mathrm{~N})$ have little impact on the reactivity value. Therefore, it can be concluded that the reaction rates discrepancies of the radiative capture, elastic scattering and inelastic scattering in the tungsten target cause the difference of the tungsten reactivity worth values. Moreover, the reactivity variation caused by the radiative capture is the largest, that is, the radiative capture reaction rates have the greatest impact on the tungsten reactivity worth.

TABLE III

CALCULATION RESULTS OF SENSITIVITY ANALYSIS FOR THE MAIN REACTION CHANNELS OF TUNGSTEN ELEMENT

\begin{tabular}{cc}
\hline Reaction channel & Reactivity $\rho$ variation $(\mathrm{mk})$ \\
\hline Radiative capture & -0.28 \\
Elastic scattering & 0.13 \\
Inelastic scattering & 0.06 \\
$\mathrm{~N}, 2 \mathrm{~N}$ & 0 \\
$\mathrm{~N}, 3 \mathrm{~N}$ & 0 \\
\hline
\end{tabular}

According to the above sensitivity analysis results, the reaction rates for the radiative capture, elastic scattering and inelastic scattering in the tungsten target were calculated with FM card in MCNP for case 1 and case 3 in Table II. Integral values of the whole neutron energy interval for the three reaction channel rates are given in the second and third columns of Table IV. From the relative difference $\mathrm{RED}\left[\mathrm{RED}=\left({ }^{\mathrm{ENDF} / \mathrm{B}-\mathrm{VII} .1}\right.\right.$ value- $\left.\left.{ }^{\mathrm{ENDF} / \mathrm{B}-\mathrm{VII} .0} \mathrm{value}\right) /{ }^{\mathrm{ENDF} / \mathrm{B}-\mathrm{VII} .0} \mathrm{value}\right]$ in the fourth column of Table IV, it can be seen that comparing with case 1 , integral value of radiation capture reaction rates in case 3 is larger, whereas that of elastic scattering and inelastic scattering rates in case 3 is smaller. And the relative difference RED of radiation capture reaction rates is maximal among the three reaction channels. Together with the results in Table III, the changes of all three reaction channel rates result in the lower tungsten reaction worth value in the case 3 and the most significant impact comes from radiation capture reaction channel. Therefore, the cross sections of non-tungsten elements mainly affect the radiative capture, elastic scattering and inelastic scattering rates, which results in the difference of tungsten reaction worth values from case 1 and case 3 . Meanwhile, the difference of radiation capture reaction rates is the main reason for the lower result in case 3.

TABLE IV

INTEGRAL VALUES OF THE REACTION RATES FOR THE MAIN REACTION CHANNELS IN THE TUNGSTEN TARGET

\begin{tabular}{cccc}
\hline Reaction channel & Case 1 & Case 3 & RED (\%) \\
\hline Radiative capture & $7.73 \mathrm{E}-06$ & $7.91 \mathrm{E}-06$ & 2.23 \\
Elastic scattering & $5.33 \mathrm{E}-05$ & $5.31 \mathrm{E}-05$ & -0.40 \\
Inelastic scattering & $7.54 \mathrm{E}-06$ & $7.51 \mathrm{E}-06$ & -0.43 \\
\hline
\end{tabular}

*Unit: number/source neutron $/ \mathrm{cm}^{3}$

In order to further analyze the influence of non-tungsten elements on main reaction channels, the whole neutrons energy region $\left(10^{-9}-10\right.$ $\mathrm{MeV}$ ) was divided into 10 energy groups in geometric progression manner of common ratio 10 . The relative differences RED of the integral values for the radiation capture, elastic scattering and inelastic scattering reaction rates were respectively calculated in each energy group for case 1 and case 3. As the results in Fig. 3(a-c) shows the maximum RED values of the radiation capture and elastic scattering between the case 1 and case 3 are both presented in the energy interval of $10^{-9}-10^{-7} \mathrm{MeV}$, whereas the RED of inelastic scattering reaction rates is very low and only found in the energy interval of $10^{-2}-1 \mathrm{MeV}$. Therefore, it can be concluded that the difference of non-tungsten elements cross sections between ENDF/B-VII.1 and ENDF/B-VII.0 most mainly results in the discrepancy of the radiation capture and elastic scattering reaction rates in the energy range of $10^{-9}-10^{-7} \mathrm{MeV}$. 


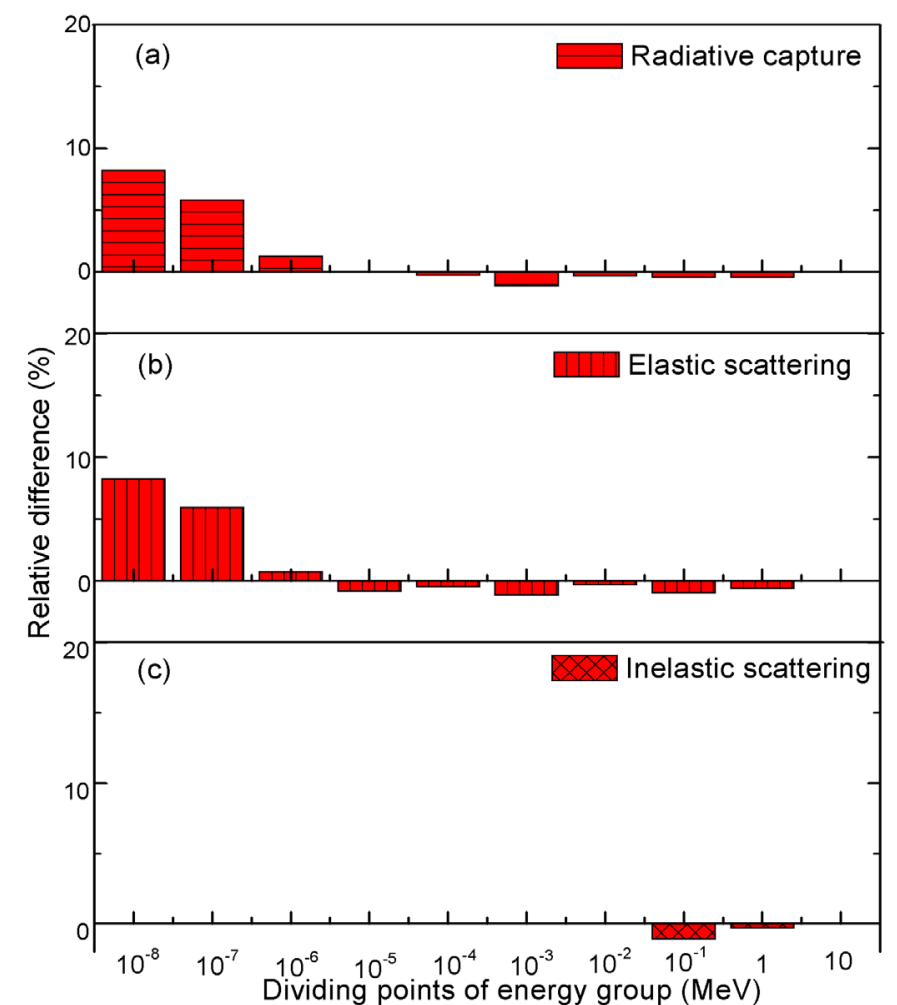

Fig. 3. Relative difference values of tungsten main reaction channel rates in the different energy groups.

\section{CONCLUSIONS AND FUTURE WORK}

The reactivity worth value of tungsten cylindrical target has been measured as $-1.234 \pm 0.114 \mathrm{mk}$ by period method on VENUS-II light water reactor at China Institute of Atomic Energy. Meanwhile, the MCNP simulations were performed with ENDF/B-VII.0, ENDF/B-VII.1, JENDL-4.0, JEFF-3.2 and CENDL-3.1, respectively. By comparing the calculated results with the experimental one, it is found that the results of ENDF/B-VII.0, JENDL-4.0 and JEFF-3.2 are higher, whereas the one of CENDL-3.1 is lower. The calculated result with ENDF/B-VII.1 has a good agreement with the experiment one and the relative deviation is less than $2 \%$. An investigation was made to mainly analyze the difference reason of the tungsten target reactivity worth values from ENDF/B-VII.1 and other libraries (ENDF/B-VII.0, JENDL-4.0 and JEFF-3.2). Through the analysis, it can be concluded that the main reason for the difference is due to the non-tungsten elements cross sections in ENDF/B-VII.1, which mainly results in the difference of the tungsten radiation capture and elastic scattering reaction rates in the energy range of $10^{-9}-10^{-7} \mathrm{MeV}$. Therefore, it is recommended to adopt ENDF/B-VII.1 to calculate the tungsten target reactivity worth. In the future, more integral experiments related to the key materials in CiADS will be done, i.e., lead, lead-bismuth and among others to satisfy the needs of the CiADS engineering design.

\section{ACKNOWLEDGEMENTS}

The work was completed under the support of Forerunner Special Foundation of Chinese academy of sciences (NO. XDA03010200). The authors express their gratitude to all members in the experimental groups of the Institute of Modern Physics, Chinese Academy of Sciences and the China Institute of Atomic Energy.

\section{REFERENCES}

[1] L. Yang, W. Zhan. A new concept for ADS spallation target: gravity-driven dense granular flow targets. Proceedings of the ThEC13 Conference, CERN, Globe of Science and Innovation, Geneva, Switzerland, 27-31 October 2013. doi: 10.1007/978-3-319-26542-1_47

[2] G. Xiao, H. Xu, S. Wang, HIAF and CiADS National Research Facilities: Progress and Prospect, Nucl Phys Rev, 34(3), 275-283(2017).

[3] X. Zhang, Y. Zhang, F. Ma and et al., Neutron production for 250 $\mathrm{MeV}$ protons bombarding on thick grain-made tungsten target, EUR PHYS J A, 51(106) (2015). doi: 10.1140/epja/i2015-15106-0

[4] Y. Ju, X. Zhang, F. Ma et al., The production of residual radionuclides by a $250 \mathrm{MeV}$ proton beam, J. Phys. G: Nucl. Part. Phys., 42(12) (2015). doi: 10.1088/0954-3899/42/12/125102

[5] Y. Li, X. Zhang, Y. Ju et al., Study of neutron spectra in a water bath from a Pb target irradiated by $250 \mathrm{MeV}$ protons, CHINESE PHYS C, 39(4) (2015). doi: 10.1088/1674-1137/39/4/044001

[6] H. Zhang, X. Zhang, F. Ma et al., Residual Nuclides Induced in $\mathrm{Cu}$ Target by a $250 \mathrm{MeV}$ Proton Beam, CHINESE PHYS LETT, 32(4) (2015). doi: 10.1088/0256-307X/32/4/042501

[7] L. Chen, F. Ma, X. Zhang et al., Spallation yield of neutrons produced in thick lead target bombarded with $250 \mathrm{MeV}$ protons, Nucl. Instrum. Methods Phys. Res., Sect. B, 342, 87 90 (2015). doi: 10.1016/j.nimb.2014.09.020

[8] W. Zhan, H. Xu, Advanced Fission Energy Program - ADS Transmutation System, CHINESE SCI BULL, 27(3): 375-381 (2012). doi: 10.3969/j.issn.1000-3045.2012.03.017

[9] A.C. Kahler, R.E. MacFarlane, R.D. Mosteller et al. ENDF/B-VII.1 neutron cross section data testing with critical assembly benchmarks and reactor experiments. NUCL DATA SHEETS, 112(12), 2997-3036 (2011). doi:10.1016/j.nds.2011.11.003

[10] S. Zhang, Z. Chen, Y. Nie et al., Measurement of leakage neutron spectra for Tungsten with D-T neutrons and validation of evaluated nuclear data, FUSION ENG DES, 92, 41-45 (2015). doi: 10.1016/j.fusengdes.2015.01.010

[11] S. Zhang, Y. Nie, J. Ren et al., Benchmarking of JEFF-3.2, FENDL-3.0 and TENDL-2014 evaluated data for tungsten with $14.8 \mathrm{MeV}$ neutrons, NUCL SCI TECH, 28:27 (2017). doi: 10.1007/s41365-017-0192-0

[12] K. Seidel, R. Eichin, U. Fischer et al. Benchmark experiments to validate the nuclear data of tungsten, FUSION ENG DES, 81, 1211-1217 (2006). doi: 10.1016/j.fusengdes.2005.09.050

[13] P. Batistoni, M. Angelone, et al. Neutron-nuclear data benchmark for copper and tungsten by slab assembly transmission experiments with D-T, FUSION ENG DES, 58-59, 617-621 (2001). doi: 10.1016/S0920-3796(01)00518-X

[14] I. Murata, T. Nishio, L. Petrizzi et al. Neutronics benchmark experiment on tungsten, J NUCL MATER, 329-333, 683-686 (2004). doi: 10.1016/j.jnucmat.2004.04.191

[15] O. Cabellos, Processing of the JEFF-3.2T3 Cross Section Library with the NJOY Code System into Various Formats for Testing Purposes (2013). JEFF Meeting, Spain, November 27-28, 2013. https://www.researchgate.net/publication/321731952

[16] M.B. Chadwick, P. Oblozinsky, M. Herman at al., ENDF/B-VII.0: next generation evaluated nuclear data library for nuclear science and technology. NUCL DATA SHEETS 107, 2931-3060 (2006). doi: 10.1016/j.nds.2006.11.001

[17] M.B. Chadwick, M. Herman, P. Oblozinsky at al., ENDF/B-VII.1 nuclear data for science and technology: cross sections, covariances, fission product yields and decay data. NUCL DATA SHEETS 112: 2887-2996 (2011). doi: 10.1016/j.nds.2011.11.002.

[18] Z. Ge, Z. Zhao, H. Xia et al., The updated version of Chinese evaluated nuclear data library (CENDL-3.1). J. Kor. Phys. Soc. 59, 1052 (2011). doi: 10.1051/ndata:07570 
[19] K. Shibata, O. Iwamoto, T. Nakagawa et al., JENDL-4.0: a new library for innovative nuclear energy systems. J. Kor. Phys. Soc., 59(23), 1046 (2011). doi: 10.3938/jkps.59.1046

[20] A. Koning, R. Forrest, M. Kellett et al., JEFF Report 21: the JEFF-3.1 Nuclear Data Library (OECD, 2006). https://www.researchgate.net/publication/267362554

[21] Q. Zhu, Q. Zhou, S. Liang et al., VENUS-II ADS Zero Power Reactor in CIAE, Proceedings of the Reactor Physics Asia Conference, Chengdu, China, 24-25 August 2017.

[22] S. Hu, R. Wang, X. Liang et al., Method and Data Processing for HTR-10 Physics Experiment, Nucl Power Eng, 23(1), 8-11 (2002).

[23] J. Khorsandi, Z. Nasr, R. Salimi et al., Heavy Water Zero Power Reactor (HWZPR) mixed core first criticality, calculation and experiments. PROG NUCL ENERG, 85, $613-616$ (2015). doi: 10.1016/j.pnucene.2015.08.007

[24] J. Wei, G. Long, Z. Qi el al. Measurement of tungsten reactivity worth on VENUS-II light water reactor and validation of evaluated nuclear data. PROG NUCL ENERG, 108, 81-88 (2018). doi: 10.1016/j.pnucene.2018.05.002

M.K. Jaradat, V. Radulovic, C.J. Park et al., Verification of MCNP6 model of the Jordan Research and Training Reactor (JRTR) for calculations of neutronic parameters, ANN NUCL ENERGY, 96, 96-103 (2016). doi: 10.1016/j.anucene.2016.06.00 